\title{
Simultaneous Spectrofluorometric Determination of Piroxicam and Pyridoxine Using Generalized Rank Annihilation Method
}

\author{
Hamid Abdollahi, ${ }^{* \dagger}$ Mohammad Hossein Sororaddin, ${ }^{* *}$ and Abdolhossein NaSeri** \\ * Department of Chemistry, Institute for Advanced Studies in Basic Sciences, Zanjan, Iran \\ **Department of Analytical Chemistry, Faculty of Chemistry, Tabriz University, Tabriz, Iran
}

\begin{abstract}
The application of generalized rank annihilation method (GRAM) to the analysis of fluorescence excitation-emission matrices of mixtures of piroxicam and pyridoxine is described. The input of GRAM consists of two bilinear data matrices, i.e. one for unknown and one for the calibration sample. The excitation wavelength range was from 290 to 340 $\mathrm{nm}$ and the emission was recorded from 370 to $560 \mathrm{~nm}$. Piroxicam and pyridoxine were determined in the concentration ranges $0.33-4.00 \mu \mathrm{g} \mathrm{ml}^{-1}$ and $0.66-8.00 \mu \mathrm{g} \mathrm{ml}^{-1}$, respectively. To check the accuracy of the proposed method, several binary synthetic mixtures and one real sample were analyzed successfully. No matrix effect was observed in mixture analysis, so a single external calibration sample was used for each analyte. The ability of GRAM to quantify the studied compounds and the comparability of GRAM results were evaluated by comparing them with those of PLS regression as a standard first-order calibration.
\end{abstract}

(Received June 29, 2005; Accepted August 5, 2005)

\section{Introduction}

One of the most serious problems that can occur in classical quantitative analysis is the presence of one or more spectral interferent-chemical species which affect the instrument response and which are unaccounted for in the calibration process. Advances in chemometric methods allow quantitative analysis in the presence of unidentified interferents if a threeway experimental data matrix is available for each sample. This property is the so-called "second-order advantage" 1 and it is based on the earlier work done in the psychometrics field..$^{2,3}$ The use of this advantage in analytical chemistry was proposed by Ho et al. ${ }^{4}$ (rank annihilation factor analysis, RAFA), for the multicomponent analysis of fluorescent mixtures using excitation-emission matrix (EEM). Later, Lorber ${ }^{5}$ and Sanchez and Kowalski ${ }^{6}$ proposed new and simple solutions for the method of Ho et al. and now it is called the generalized rank annihilation method (GRAM).

At present, analytical chemistry laboratories have instrumentation that easily generates multidimensional data structures of experimental data for each sample. Fluorescence is a particularly interesting technique because it allows, in a very straightforward manner, the acquisition of this type of information, of which the most typical example is the excitation-emission matrix (EEM). Several algorithms that use second-order data are described in the literature. ${ }^{7-9}$ Of these, the generalized rank annihilation method (GRAM) $)^{6,11,12}$ has interesting properties since it only requires two data matrices for quantification: one from the calibration sample and the other from the unknown sample. Recently, it has been proved that GRAM predicts results that are similar to those from the parallel factor analysis (PARAFAC) when only two samples are used, ${ }^{9}$ so both methods can be useful as calibration methods for second-order data. But, unlike

† To whom correspondence should be addressed.
PARAFAC, GRAM is a non-iterative method which tries to implement the second-order advantage. Second-order calibration using GRAM has several distinct advantages. ${ }^{13}$ The most pronounced advantage is that it makes possible the quantification of the analyte(s) of interest in samples containing interferents. Those interferents can be completely unknown, that is, it is not necessary that they have been incorporated in the standards used for calibration. Another advantage is that it is possible to recover the individual instrument responses of the analyte(s) of interest, e.g., the excitation and emission spectra can be obtained for the individual analyte(s). This makes it possible to perform qualitative analysis and to check the identity of the analytes. The Kowalski group has proposed the trilinear decomposition (TLD) method. ${ }^{14,15}$ In this work we focused our attention on the ability of GRAM for analyzing the second-order data.

Fluorescence spectroscopy is a versatile tool mainly used because of its selectivity and sensitivity. Fluorescence spectra can be recorded in difference modes such as emission, excitation, synchronous and excitation-emission matrix (EEM). The arrangement of fluorescence data in an array (i.e. first-order data) can be analyzed with first-order calibration methods such as partial least squares (PLS) regression. This approach has been successfully applied to simultaneous mixture analysis. ${ }^{16,17}$ However, first-order calibration methods require that both unknown and standard samples have the same chemical and physical characteristics, i.e. all detectable species present in the unknown samples, including analytes and interferences, must also be present in the standard samples. The most expensive step in first-order multivariate calibration methods is the preparation and analysis of the large number of standards that have to be used for calibration. Alternatively, fluorimetric data can be arranged in data matrices (i.e. second order data) for analysis with second-order calibration methods, which may take advantage of features of both excitation and emission spectra of the compound studied. The use of fluorescence excitation-emission matrices (EEMs) for the two orders of data is advantageous 
<smiles>CN1C(C(=O)Nc2ccccn2)=C(O)c2ccccc2S1(=O)=O</smiles>

Piroxicam<smiles>Cc1ncc(CO)c(CO)c1O</smiles>

Pyridoxine

Scheme 1

because (i) the measurements can be made on a single instrument with consistent channel registration, (ii) EEMs exhibit good sensitivity, selectivity, and bilinearity, and (iii) a range of options is available for the third order. Variation in the sample composition is the most common approach to introducing the third order in fluorescence EEMs. ${ }^{18,19}$

Piroxicam (PX), 4-hydroxy-2-methyl- $\mathrm{N}$-(2-pyridyl)-2H-1,2benzothiazine-3-carboamide-1,1-dioxide (Scheme 1), is a nonsteroidal anti-inflammatory agent which is widely used in the treatment of rheumatic diseases. ${ }^{20}$ The literature reports several analytical methods for the determination of PX in pharmaceutical samples and biological fluids ${ }^{21-26}$ including spectrofluorimetry methods due to high sensitivity and selectivity. ${ }^{27-30}$ In pharmaceutical preparations, PX is frequently combined with some vitamins of the B complex. Among B complex vitamins, pyridoxine, PY, (2- methyl-3-hydroxy-4,5-bis(hydroxyl-methyl)pyridine (Scheme 1)) is one of the members of the vitamin B6 group, and possesses a significant native fluorescence in solution, which often interferes in the spectrofluorimetric determination of PX. ${ }^{31}$ Because PY is essential for both protein and red blood cell metabolism, ${ }^{32}$ several methods have been developed for its determination, including fluorescent ones. ${ }^{33,34}$ Escander et al. ${ }^{31}$ determined PX and PY in pharmaceutical preparations by fluorimetry after separating them by solid-phase extraction. To the best of our knowledge, there is not any report about spectrofluorimetric simultaneous determination of these two compounds in pharmaceutical preparations without separation.

The aim of this work is the application of GRAM to simultaneous determination of PX and PY in pharmaceutical formulations based on their native fluorescence. Calibration is carried out using an external standard for each analyte. Firstorder data, emission spectra, were analyzed with partial least squares regression as a first-order calibration method. The comparison of the results obtained by the two models was also an objective of this work.

\section{Experimental}

\section{Instrumentation}

All fluorescence measurements were done on a Cary Eclipse Fluorescence Spectrophotometer (Varian, Australia) equipped with a Xenon lamp pulsed at $80 \mathrm{~Hz}$. The measurements were done using $1.00 \mathrm{~cm}$ quartz cell and slits widths of $5 \mathrm{~nm}$ for both excitation and emission monochromators. The scan rate of the monochromator was maintained at $120 \mathrm{~nm} / \mathrm{min}$ in recording of emission and excitation-emission spectra. The $\mathrm{pH}$ measurements were carried out with a Metrohm $713 \mathrm{pH}$ meter (Herisau, Switzerland) equipped with a glass electrode.

A Fungilab ultrasonicator was used for sonication of pharmaceutical solutions and a Bifuge Primo Heraeus centrifuge instrument was used for centrifuging of pharmaceutical solutions.

Data acquisition and data analysis were performed with Matlab 6.5. The m-file for performing of GRAM was written

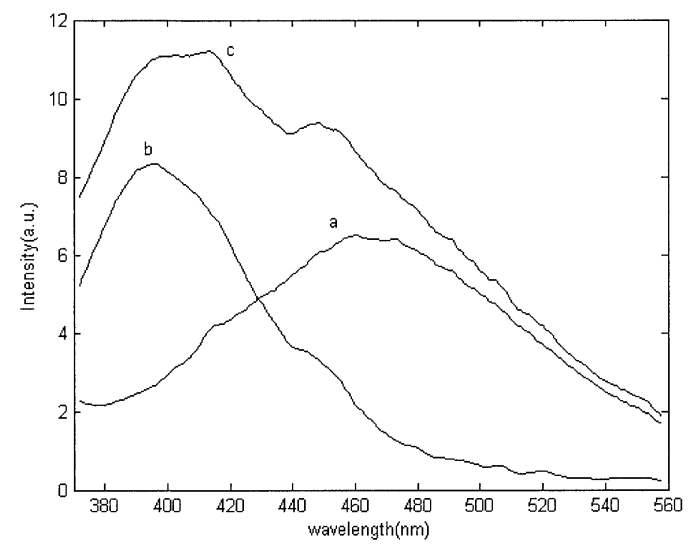

Fig. 1 Fluorescence spectra of $4.0 \mu \mathrm{g} \mathrm{ml}^{-1}$ pyridoxine (a), $2.0 \mu \mathrm{g}$ $\mathrm{ml}^{-1}$ piroxicam (b) and mixture of them (c). $\lambda_{\mathrm{ex}}, 320 \mathrm{~nm} ; \mathrm{pH}, 1(\mathrm{HCl}$, $0.1 \mathrm{M}$ ); slits, $5 \mathrm{~nm}$.

locally. PLS analyses were carried out by using PLS-Toolbox software version 2.0. ${ }^{10}$

\section{Materials and reagents}

Analytical-reagent grade chemicals were employed in all experimens. PX was obtained from Sigma (USA). PY, $\mathrm{NH}_{3}$, $\mathrm{HCl}$ and $\mathrm{NaCl}$ were purchased from Merck (Darmstadt, Germany). A stock solution of PX $\left(300 \mu \mathrm{g} \mathrm{ml}^{-1}\right)$ was prepared by dissolving $30.0 \mathrm{mg}$ of PX in $2-3 \mathrm{ml}$ of concentrated $\mathrm{NH}_{3}$. This was then diluted with distilled water and 2-3 $\mathrm{ml}$ of concentrated $\mathrm{HCl}$ was added. Finally the mixture was diluted to the mark in a $100.0 \mathrm{ml}$ volumetric flask. An aqueous stock solution of PY $\left(400 \mu \mathrm{g} \mathrm{ml}^{-1}\right)$ was obtained by dissolving of this vitamin in $100.0 \mathrm{ml}$ of water. $\mathrm{A} \mathrm{HCl}(0.1 \mathrm{M}) / \mathrm{NaCl}(0.1 \mathrm{M})$ solution was also employed as a buffer.

\section{Treatment of sample}

About 2.0 g gel (0.5\% piroxicam, Razak Co., Tehran, Iran) was accurately weighed and dissolved in $15 \mathrm{ml} \mathrm{NH}_{3}$ while stirring; this mixture was sonicated for $5 \mathrm{~min}$ and then diluted to the mark in a $100.0 \mathrm{ml}$ volumetric flask.

Ten tablets of pyridoxine (40 mg B6 vitamin, Iran Hormon Co., Tehran, Iran) were weighed in order to find the average mass of each tablet. Then the content was powdered and mixed. A portion of the powder equivalent to about $40 \mathrm{mg}$ of PY was weighed accurately, dissolved in about $40 \mathrm{ml}$ water and sonicated for $5 \mathrm{~min}$. The resulting solution was centrifuged at $2500 \mathrm{rpm}$ for $5 \mathrm{~min}$ and filtered into a $100 \mathrm{ml}$ volumetric flask. The residue was washed several times with water and the solution was diluted to the mark with the same solvent.

\section{Procedure and acquisition of data sets}

A $3.0 \mathrm{ml}$ portion of buffer was transferred to a quartz cell and needed amounts of standards (PX and PY) or sample solutions were to added it by micropipet syringe, then the solution was mixed and spectra were recorded.

First order data (emission spectra) were recorded in the range of 370 to $560 \mathrm{~nm}$ with $1 \mathrm{~nm}$ intervals using an excitation wavelength of $320 \mathrm{~nm}$. Excitation-emission matrices (secondorder data) were measured every $2 \mathrm{~nm}$ in the emission range $370-560 \mathrm{~nm}$ and every $5 \mathrm{~nm}$ in the excitation range $290-340$ $\mathrm{nm}$, making a total of $92 \times 11=1012$ data points. 
Table 1 Analytical figures of merit for both analytes

\begin{tabular}{ccl}
\hline Pyridoxine & Piroxicam & \multicolumn{1}{c}{ Parameter } \\
\hline $0.66-8.00$ & $0.33-4.00$ & Dynamic linear range $\mu \mathrm{g} \mathrm{ml}^{-1}$ \\
0.9992 & 0.9975 & Correlation coefficient \\
0.21 & 0.22 & Limit of detection $/ \mu \mathrm{g} \mathrm{ml}^{-137}$ \\
3.04 & 3.50 & $\mathrm{RSD} \%$ \\
$y=1.77 x+1.13$ & $y=2.30 x+1.55$ & $\begin{array}{l}\text { Equation of calibration curve } \\
\text { (fluorescence intensity versus }\end{array}$ \\
& & $\mu \mathrm{g} \mathrm{ml}^{-1}$ of analyte) \\
\hline
\end{tabular}

a. In order to obtain the calibration curves of drugs, we measured the fluorescence intensity at 465 and $400 \mathrm{~nm}$ for PX and PY, respectively, while the excitation wavelength for both was $320 \mathrm{~nm}$.

\section{Results and Discussion}

\section{Fluorescence characteristics of drugs: optimization}

PX is soluble in alkaline solution but it only emits fluorescence in acid solutions. We found that a suitable procedure was to dissolve it in $\mathrm{NH}_{3}$, adding $\mathrm{HCl}$ in order to register the fluorescence spectrum. PY is soluble in water therefore it is dissolved in water.

The influence of $\mathrm{pH}$ on the fluorescence intensity of each drug was studied by adding small volumes of a dilute solution of $\mathrm{HCl}$ and $\mathrm{NaOH}$ to adjust the $\mathrm{pH}$. It is observed that PX only emits fluorescence in low $\mathrm{pH}$, acidic media. PY emits fluorescence in all $\mathrm{pH}$ values and its fluorescence intensity in acidic media is lower than in basic media, but it is comparable with the fluorescence of $\mathrm{PX}$. $\mathrm{HCl} 0.1 \mathrm{M}$ was chosen as optimum value for subsequent experiments in order to ensure maximum signal for PX. The use of $\mathrm{HNO}_{3}$ had poor analytical characteristics for quantitation of PX.

Figure 1 shows the solution-fluorescence spectra of PX and PY in acidic media at concentrations similar to those found in typical pharmaceutical compositions. All fluorescence spectra were recorded at the maximum excitation wavelength of PX $(320 \mathrm{~nm})$ to favor the fluorescence enhancement of the weaker emitter. The spectral overlapping from PY is clearly observed and the direct determination of PX at parts-per-million ( $\mathrm{ppm}$ ) level would not be possible in the presence of PY. So, the application of chemometric methods for the simultaneous determination of PX and PY without separation is necessary.

\section{Individual calibrations}

Individual calibration curves were constructed with several points as fluorescence intensity versus drug concentration in the range $0.3-4.0 \mu \mathrm{g} \mathrm{ml}^{-1}$ for PX and $0.7-8.0 \mu \mathrm{g} \mathrm{ml}^{-1}$ for PY and evaluated by linear regression. In order to obtain the calibration curves of drugs, we measured the fluorescence intensity at 465 and $400 \mathrm{~nm}$ for PX and PY respectively, while the excitation wavelength for both was $320 \mathrm{~nm}$. The repeatability, expressed by relative standard deviation, the linear range, the sensitivity, the detection limits and the correlation coefficients were obtained for PX and PY and they are presented in Table 1.

\section{Partial least squares}

The arrangement of fluorescence data in an array (i.e. firstorder data) was analyzed with partial least square regression as a first-order calibration method. PLS was selected as a standard multivariate calibration method for comparing and evaluating the results of GRAM as a second-order method. However, firstorder calibration methods require that both unknown and standard samples have the same chemical and physical
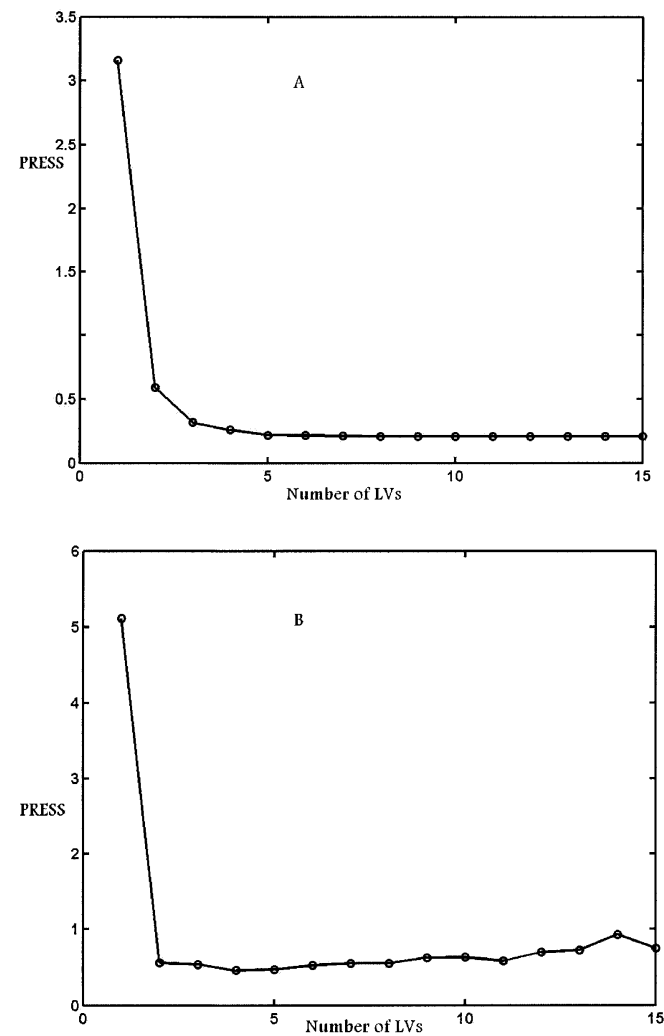

Fig. 2 Cross validation plots for (A) piroxicam (B) pyridoxine.

characteristics. The most expensive step in first-order multivariate calibration methods is the preparation and analysis of the large number of standards that have to be used for calibration.

Twenty binary mixtures were selected as the calibration set. The composition of the samples was randomly designed in order to obtain non-correlated concentration profiles. The correlation between concentration vectors can be minimized if the correlation coefficient matrix is considered as the criterion. The obtained model in the calibration step was validated with 9 synthetic mixture sets containing the drugs in different proportion ranges according to the compositions of considered drugs in several pharmaceutical formulations that were randomly selected. Fluorescence emission data from calibration set were taken between 370 and $560 \mathrm{~nm}$. The concentration range of analytes in all synthetic samples was $0.3-4.0 \mu \mathrm{g} \mathrm{ml}^{-1}$ for PX and $0.6-8.0 \mu \mathrm{g} \mathrm{ml}^{-1}$ for PY.

The selection of the optimum number of factors was estimated by cross-validation, leaving out one sample at a time. ${ }^{35}$ The predicted concentration of the analytes in each sample was then compared with the known concentration of them in the respective sample and the prediction error sum-of-square (PRESS) was calculated. This parameter was calculated each time adding a new factor to the model and is shown in Fig. 2. A plot of the PRESS against the number of factors for each individual component indicates a minimum value for an optimal number of factors. For finding the smallest model (fewest numbers of factors) the F statistics was used to carry out the significance determination. ${ }^{36}$ It was found that the optimum number of factors was three for both PX and PY (Fig. 2). The results obtained from spectrofluorimetric simultaneous analysis of PX and PY by PLS method are given in Table 2. The values of root mean square difference (RMSD), the square of the correlation coefficient obtained when plotting actual versus 
Table 2 Statistical parameter for PX and PY in validation set of PLS model

\begin{tabular}{lcc}
\hline & Piroxicam & Pyridoxine \\
\hline $\mathrm{RMSD}^{\mathrm{a}}$ & 0.11 & 0.19 \\
$\mathrm{REP}^{\mathrm{b}}$ & $5.38 \%$ & $3.69 \%$ \\
$R^{2 \mathrm{c}}$ & 0.9953 & 0.9928 \\
\hline
\end{tabular}

a. RMSD calculated according to: $\mathrm{RMSD}=\left(\left(\sum\left(C_{\text {real }}-C_{\text {found }}\right)^{2}\right) / \Sigma\right.$ $\left.\left(C_{\text {found }}\right)^{2}\right)^{1 / 2}$.

b. REP calculated according to: $\operatorname{REP}=100 \times\left(\left(\sum\left(C_{\text {real }}-C_{\text {found }}\right)^{2}\right) / n\right)^{1 / 2}$.

c. Correlation coefficient for plotting the $C_{\text {real }}$ versus $C_{\text {found. }}$.

predicted concentration $\left(R^{2}\right)$, and the relative error of prediction (REP) for each component in nine synthetic samples are included in order to give an indication both of the average error in analysis and the quality of fit of all data to a straight line.

\section{Generalized rank annihilation method}

The input of a GRAM calculation consists of $J_{1} \times J_{2}$ bilinear data matrices,

$$
\begin{aligned}
& \mathbf{D}_{\text {cal }}=\mathbf{X C}_{\text {cal }} \mathbf{Y}^{\mathrm{T}}+\mathbf{E}_{\mathrm{cal}} \\
& \mathbf{D}_{\mathrm{un}}=\mathbf{X C}_{\mathrm{un}} \mathbf{Y}^{\mathrm{T}}+\mathbf{E}_{\mathrm{un}}
\end{aligned}
$$

Here $\mathbf{D}_{\text {cal }}$ and $\mathbf{D}_{\text {un }}$ are measured for the calibration and unknown sample; $\mathbf{X}\left(J_{1} \times K\right)$ and $\mathbf{Y}\left(J_{2} \times K\right)$ contain the excitation and emission spectral profiles ( $K$ is the number of constituents present in both samples); $\mathbf{C}_{\text {cal }}$ and $\mathbf{C}_{\text {un }}$ are $K \times K$ diagonal matrices of concentrations (with zero diagonal elements for the analytes that are absent), and $\mathbf{E}_{\text {cal }}$ and $\mathbf{E}_{\text {un }}$ are $J_{1} \times J_{2}$ error matrices.

GRAM is a principal component analysis (PCA)-based curve resolution method: In the first step, the sum matrix $\mathbf{Q}=\mathbf{D}_{\text {cal }}+$ $\mathbf{D}_{\mathrm{un}}$ is decomposed by singular value decomposition as $\mathbf{Q}=$ $\mathrm{USV}^{\mathrm{T}}$ to get truncated orthogonal factor matrices $\mathbf{U}\left(J_{1} \times A\right)$ and $\mathbf{V}\left(J_{2} \times A\right)$. The number of factors $A$ is an estimate of $K$. In the second step, an eigenvalue problem is solved, $\left(\mathbf{S}^{-1} \mathbf{U}^{\mathrm{T}} \mathbf{D}_{\text {un }} \mathbf{V}\right) \mathbf{T}=$ $\mathrm{T} \Lambda$. $\mathbf{D}_{\text {un }}$ is projected to the factor space defined by $\mathbf{Q}$ to obtain the eigenvector matrix $\mathbf{T}$ and the matrix of eigenvalues $\Lambda$. In the last step, the $\mathbf{X}$ and $\mathbf{Y}$ matrices are estimated by applying the transformation matrix $\mathbf{T}$ on $\mathbf{U}$ and $\mathbf{V}$, respectively. The prediction for analyte of interest is obtained by combining the measured concentration in the calibration sample, $c_{\text {cal }}$, and the associated eigenvalue, $\lambda$, as

$$
\mathbf{C}_{\mathrm{un}}=c_{\mathrm{cal}} \lambda /(1-\lambda)
$$

This calculation requires $c_{\text {cal }}$ and $\lambda$ to be matched, which in practice is achieved by comparing the estimated spectral profiles (appropriate column of $\mathbf{X}$ and $\mathbf{Y}$ ) with reference profiles.

Figure 3 shows the EEMs for $1.98 \mu \mathrm{g} \mathrm{ml}^{-1}$ and $4.61 \mu \mathrm{g} \mathrm{ml}^{-1}$ of PX and PY respectively, which were selected as standards for GRAM analysis. In order to test the performance of the proposed method, several synthetic binary mixtures of drugs in various concentration proportions were analyzed by using the GRAM algorithm. The results of estimating the concentration of both drugs for 5 binary mixtures are presented in Table 3 . The compositions of synthetic samples also are selected according to the proportions of drugs in several pharmaceutical formulations. As can be seen, the amounts added and found were consistent for the tested mixtures. The results can be

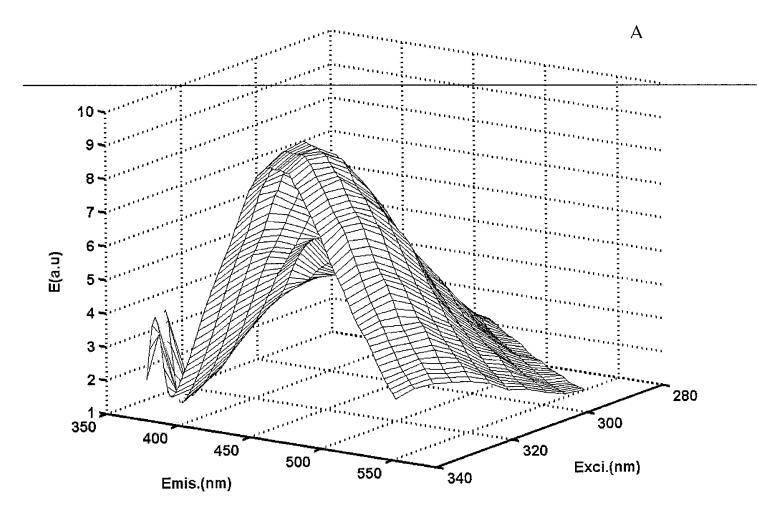

B

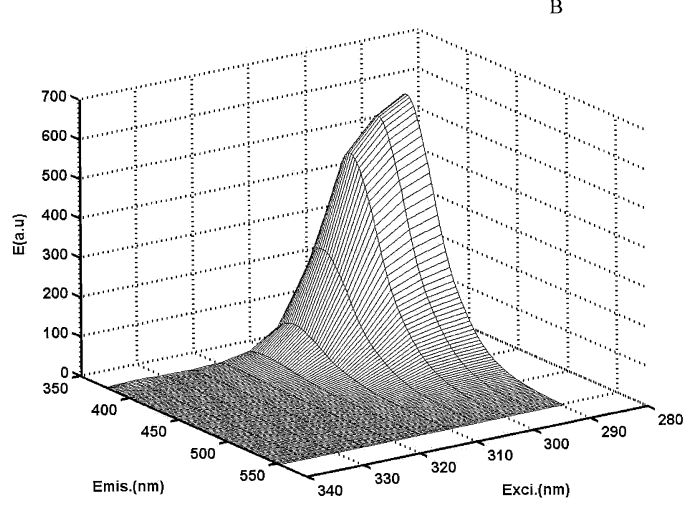

Fig. 3 Excitation-emission matrix spectra of (A) piroxicam (1.98 $\left.\mu \mathrm{g} \mathrm{ml}^{-1}\right)$, (B) pyridoxine $\left(4.61 \mu \mathrm{g} \mathrm{ml}^{-1}\right)$ in $0.1 \mathrm{M} \mathrm{HCl}$.

considered satisfactory, keeping in mind the strong spectral overlapping for PX and PY and the lower fluorescence signal from PX compared with that from PY.

In the presence of matrix effects which could be exist in very complex samples, GRAM can be combined with a standard addition method. In this work, the obtained results from standard addition method and using the external standards had not any significant difference even for real samples. Therefore, the external standard calibration is proposed for simultaneous determination of PX and PY in pharmaceutical formulations. External standard calibration is simpler to implement because a single standard set is used for all unknown samples.

\section{Real sample}

To examine the applicability of the methods, we used PLS and GRAM in the simultaneous determination of the two analytes in pharmaceutical formulations. Although there is a pharmaceutical formulation containing both considered drugs, we could not prepare that in our country. The available pharmaceutical test did not contain the two analytes together. Therefore, the samples prepared from the piroxicam gel and tablet of pyridoxine were mixed and used as a real sample. According to labels of used gel and tablets the artificial real sample was prepared in the same proportion as that of a commercial formulation. ${ }^{31}$ The results for both analytes are shown in Table 4. Good recovery results for both drugs show that excipients do not interfere in the determination of PX and PY. In comparing of two chemometric methods for simultaneous determining PX and PY in pharmaceutical preparation, one may note that the GRAM results are as satisfactory as those furnished by PLS. Further, from the points 
Table 3 Results for analysis of five synthetic samples by GRAM

\begin{tabular}{ccccccc}
\hline \multirow{2}{*}{ Sample } & \multicolumn{2}{c}{ Piroxicam $/ \mu \mathrm{g} \mathrm{ml}^{-1}$} & & \multicolumn{2}{c}{ Pyridoxine $/ \mu \mathrm{g} \mathrm{ml}^{-1}$} \\
\cline { 2 - 3 } \cline { 5 - 6 } \cline { 5 - 6 } & Added & Found & & Added & Found \\
\hline 1 & 1.95 & 2.12 & & 3.21 & 3.04 \\
2 & 3.88 & 3.66 & & 3.84 & 3.48 \\
3 & 2.88 & 2.85 & & 1.59 & 1.77 \\
4 & 2.87 & 2.92 & & 2.91 & 3.06 \\
5 & 1.45 & 1.77 & & 2.93 & 2.92 \\
RMSD, \% & & 6.98 & & & \multicolumn{3}{c}{7.09} \\
\hline
\end{tabular}

Table 4 Results for analysis of drugs contain piroxicam (gel) and pyridoxine (tablet) by two chemometrics method

\begin{tabular}{lccccc}
\hline & \multicolumn{2}{c}{ Piroxicam } & & \multicolumn{2}{c}{ Pyridoxine } \\
\cline { 2 - 3 } \cline { 5 - 6 } & PLS & GRAM & & PLS & GRAM \\
\hline Found $^{\mathrm{a}}$ & $0.52 \%$ & $0.52 \%$ & & $43.03 \mathrm{mg}$ & $38.86 \mathrm{mg}$ \\
& $(0.5 \%)$ & $(0.5 \%)$ & & $(40 \mathrm{mg})$ & $(40 \mathrm{mg})$ \\
Recovery, $\%^{104}$ & 104 & 104 & & 108 & 97 \\
RMSD $^{\mathrm{b}}$ & 0.14 & 0.25 & & 0.35 & 0.25 \\
\hline
\end{tabular}

a. Data in parentheses are the label values of drugs.

b. RMSD calculated according to: $\mathrm{RMSD}=\left(\left(\sum\left(C_{\text {real }}-C_{\text {found }}\right)^{2}\right) / \Sigma\right.$ $\left.\left(C_{\text {found }}\right)^{2}\right)^{1 / 2}$.

of view of cost and time required for calibration, the use of the GRAM is significantly better to implement than PLS, because only one calibration solution for each analyte was used.

\section{Conclusions}

The potentials of two chemometric methods for resolving overlapped fluorescence spectra in binary mixtures of two important active principles: piroxicam and pyridoxine have been demonstrated. The analysis can be carried out with avoiding preliminary separation steps.

Two types of fluorescence spectra data were used: emission spectra as first-order data and excitation-emission spectra as second-order data. PLS and GRAM were used for analyzing of first-order data and second-order data, respectively. The generalized rank annihilation method proved to be useful for quantifying the drug constituents in complex real samples. It requires only one standard sample for quantification, which make it easier than a first-order method such as PLS.

The proposed method for simultaneous spectrofluorimetric determination of PX and PY is a rapid one-step procedure which only requires the dissolution of the sample and the acquisition of excitation-emission spectra, so it is a simple, inexpensive and fast procedure which does not need a previous separation of the analytes nor other previous sample treatments.

\section{References}

1. K. Booksh and B. R, Kowalski, Anal. Chem., 1994, 66, 782A.

2. H. G. Law, C. W. Snider Jr., J. A. Hattie, R. P. McDonald (ed.), "Research Methods for Multimode Data Analysis", 1984, Praeger, New York.

3. R. Coppi and S. Bolasco (ed.), "Multiway Data Analysis",
1989, North-Holland, Amsterdam,

4. C. N. Ho, G. D. Christian, and E. R. Davidson, Anal. Chem., 1978, 50, 1108.

5. A. Lorber, Anal. Chim. Acta, 1984, 164, 293.

6. E. Sanchez and B. R. Kowalski, Anal. Chem., 1986, 58, 496.

7. A. De Juan and R. Tauler, J. Chemom., 2001, 15, 749.

8. N. M. Faber, R. Bro, and P. K. Hopke, Chemom. Intel. Lab. Syst., 2003, 65, 119.

9. N. M. Faber, Anal. Bioanal. Chem., 2002, 372, 683.

10. B. M. Wise and N. B. Gallagher, "PLS Toolbox for Use with MATLAB", 1997, Eigenvector Reseach, Manson, WA.

11. N. M. Faber, L. M. C. Buydens, and G. Kateman, J. Chemom., 1994, 8, 181.

12. E. Comas, R. A. Gimeno, J. Fere, R. M. Marce, F. Burrull, and F. X. Rius, J. Chromatogr., A, 2003, 988, 277.

13. A. K. Smilde, R. Tauler, J. Saurrina, and R. Bro, Anal. Chim. Acta, 1999, 398, 237.

14. E. Sanchez and B. R. Kowalski, J. Chemom., 1990, 4, 29.

15. K. S. Booksh, Z. Lin, Z. Wang, and B. R. Kowalski, Anal. Chem., 1994, 66, 2561.

16. H. Abdollahi, Anal. Chim. Acta, 2001, 442, 327.

17. H. Abdollahi and L. Bagheri, Anal. Chim. Acta, 2004, 514, 211.

18. P. D. Wentzell, S. S. Nair, and R. D. Guy, Anal. Chem., 2001, 73, 1408.

19. J. Saurrina, C. Leal, R. Compano, M. Granados, R. Tauler, and M. D. Prat, Anal. Chim. Acta, 2000, 409, 237.

20. Y. H. Chang, S. H. Dromgoole, in "Drugs for Rheumatic Disease", ed. H. E. Paulas, D. E. Furst, and H. S. Dromgoole, 1978, Chap. 18, Churchil Livingstone, New York.

21. E. Hackmann, E. Gianotto, and M. Santoro, Anal. Lett., 1993, 26, 259.

22. A. J. Nepote, L. Vwra-Candiotti, M. R. Williner, P. C. Damiani, and A. C. Olivieri, Anal. Chim. Acta, 2003, 489, 77.

23. B. S. Nagaralli, J. Seetharamappa, and M. B. Melwanki, J. Pharm. Biomed. Anal., 2002, 29, 859.

24. C. Sanchez Pedreno, M. S. Garcia, M. I. Albero, and J. Rodriguez, J. Pharm. Biomed. Anal., 1993, 11, 933.

25. A. Augerinos, S. Axalis, J. Dragasti, and T. Karidas, J. Chromatogr., B, 1995, 673, 142.

26. K. Nikolic, M. Bogavac, and L. Arsenijevic, Farmaco, 1993, 48, 1131.

27. J. A. Arancibia and G. M. Escandar, Talanta, 2003, 60, 1113.

28. G. M. Escandar, Analyst, 1999, 124, 587.

29. J. L. Manzoori and M. Amjadi, Microchim. Acta, 2003, 143, 39.

30. P. C. Damini, M. Bearzotti, M. Cabezon, and A. C. Olivieri, J. Pharm. Biomed. Anal., 1998, 17, 223.

31. G. M. Escandar, A. J. Bystol, and A. D. Compiglia, Anal. Chim. Acta, 2002, 466, 275.

32. D. A. Bender, Proc. Nutr. Soc., 1994, 53, 625.

33. L. Garcia, S. Blazquez, M. P. San Andres, and S. Vera, Anal. Chim. Acta, 2001, 434, 193.

34. N. R. Martos, A. M. Diaz, A. Navalon, I. De Orbe Paya, and L. F. Capitan Vallvey, J. Pharm. Biomed. Anal., 2000, 23, 837.

35. S. Wold, Technometrics, 1978, 20, 397.

36. D. M. Haaland and E. V. Thomas, Anal. Chem., 1988, 60, 1193.

37. J. N. Miller and J. C. Miller, "Statistics and Chemometrics for Analytical Chemistry", 4th ed., 2000, Prentice Hall, Edinburgh Gate. 